\title{
COHOMOLOGY OF ASSOCIATIVE TRIPLE SYSTEMS
}

\author{
RENATE CARLSSON
}

\begin{abstract}
In the following a cohomology for associative triple systems is defined. The classical corollaries can be extended to the associative triples.
\end{abstract}

1. Introduction. One distinguishes two associative triple systems. The associative triples of the first kind or ternary rings were introduced by Lister [6]. Those of the second kind or Hestenes ternary rings were first investigated by Hestenes and as special alternative triples by Loos [7]. Typical examples for the latter are the $p \times q$ matrices in an associative algebra with an involution, the triple product given by $(x, y, z) \mapsto x x^{t} z,{ }^{t} \bar{y}$ the transposed-conjugate matrix.

In this note we define a canonic cohomology for associative triples. Cohomologies are well known for certain algebras and Lie triples [2]. The corollaries for associative triples are analogous to the classical results.

In the following $K$ is a commutative and associative ring with unit 1 , the modules taken unitary over $K$. If $K$ is explicitly assumed as a field then the dimensions over $K$ are finite.

2. Definitions. The universal $(j-)$ imbedding. A $K$-module $A$ with a trilinear inner composition $A \times A \times A \rightarrow A$, with $(x, y, z) \mapsto\langle x y z\rangle$, is an associative triple system of the 1 st kind if

$$
\langle\langle x y z\rangle u v\rangle=\langle x\langle y z u\rangle v\rangle=\langle x y\langle z u v\rangle\rangle
$$

for $x, y, z, u, v \in A . A$ is an associative triple of the 2 nd kind, when

$$
\langle\langle x y z\rangle v\rangle=\langle x\langle u z y\rangle v\rangle=\langle x y\langle z u v\rangle\rangle \text {. }
$$

A trimodule $M$ over a triple system $A$ is a $K$-module $M$ together with 3 trilinear compositions $A \times A \times M \rightarrow M$, the products denoted $\langle x y m\rangle$, $\langle x m y\rangle,\langle m x y\rangle$, where $m \in M$. The split null extension or semidirect sum $A+M$ of $M$ over $A$ is the $K$-module direct sum with

$$
\left\langle\left(x+m_{1}\right)\left(y+m_{2}\right)\left(z+m_{3}\right)\right\rangle:=\langle x y z\rangle+\left\langle m_{1} y z\right\rangle+\left\langle x m_{2} z\right\rangle+\left\langle x y m_{3}\right\rangle
$$

for $x, y, z \in A, m_{i} \in M, i=1,2,3$. A trimodule $M$ is associative of the 1 st kind over $A$ if the semidirect sum is an associative triple of the lst kind, equally for the 2 nd kind. In the following $A$ always denotes an associative triple, and $M$ an associative trimodule over $A$ of the same kind if nothing

Received by the editors September 16, 1975.

AMS (MOS) subject classifications (1970). Primary 16A78, 18H15, 18H40; Secondary 08A25, 16A21, 16A28.

Key words and phrases. Cohomology groups of associative triple systems, universal ( $j$-) imbedding, Whitehead lemmata, Wedderburn principal theorem, invariant submodules, trimodules. 
other is stated, where associative means associative of the 1st or 2 nd kind. For $K$-modules $N, P$ let $N \oplus P$ denote the $K$-module direct sum.

Let $B$ be an associative algebra, the multiplication designed by " $\circ$ ", and $\iota$ : $A \rightarrow B$ a $K$-module monomorphism. $(B, \iota)$ is an imbedding of an associative triple $A$ of the 1 st kind if $B=\iota A+\iota A \circ \iota A$ with $\iota x \circ \iota y \circ \iota z=\iota\langle x y z\rangle, x, y$, $z \in A$, and the imbedding is direct if the sum is direct [6]. $(B, \iota)$ is universal if for each homomorphism $f: A \rightarrow A^{\prime}$ onto a triple $A^{\prime}$, and any imbedding ( $B^{\prime}$, $\kappa)$ of $A^{\prime}$ with monomorphism $\kappa$ there exists a unique algebra homomorphism $\tilde{f}: B \rightarrow B^{\prime}$ onto $B^{\prime}$ so that the diagram

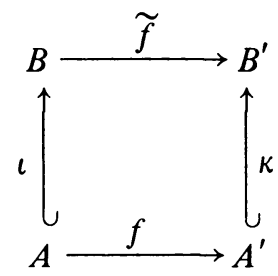

is commutative, $\hookrightarrow$ denoting a $K$-module monomorphism.

Let $A$ be associative of the 2nd kind, $j$ an involution of $B$ with $\overline{\imath A}:=j(\iota A)$, $\bar{b}:=j(b), b \in B .(B, \imath, j)$ is a j-imbedding of $A$ if $B=\imath A+\overline{\imath A}+\iota A \circ \overline{\imath A}$ $+\overline{\iota A} \circ \iota A$ with $\iota A \circ \iota A=\{0\}$ and $\iota x \circ \overline{\iota y} \circ \iota z=\iota\langle x y z\rangle$ for $x, y, z \in A$. The $j$-imbedding is direct if the above sum is direct. $(B, \iota, j)$ is universal if for any homomorphism $f$ onto $A^{\prime},\left(B^{\prime}, \kappa, j^{\prime}\right)$ a $j^{\prime}$-imbedding of $A^{\prime}$, there exists a unique homomorphism $\tilde{f}: B \rightarrow B^{\prime}$ so that the above diagram is commutative and $j^{\prime} \tilde{f}=\tilde{f}$. The standard imbedding of $A$ is a direct imbedding if $A$ is associative of the 1 st kind [6], and it contains a direct $j$-imbedding otherwise, as is easily noticed (cf. [7]). One immediately has

THEOREM 1. Let $A$ be an associative triple over $K$. Then there exists a universal imbedding $(U(A), \iota)$, respectively a universal j-imbedding $(U(A), \iota, j)$ of $A$. If $(V(A), \kappa)$, respectively $\left(V(A), \kappa, j^{\prime}\right)$, is a universal $\left(j^{\prime}-\right)$ imbedding too, then there is a unique isomorphism $g$ of $U(A)$ onto $V(A)$ with $\kappa=g$.

Proof. Let $T(A):=K \cdot 1 \oplus T^{*}(A)$ be the tensor algebra of the $K$-module $A$ with $T^{*}(A):=\bigoplus\left(\otimes^{n} A\right), n \in \mathbf{N}$. Then $T(A)$ is a universal enveloping associative algebra for $A$ with unit 1. We construct $U(A)$ as a factor algebra: In case of the 2nd kind let $\bar{A}$ be a $K$-module isomorphic to $A$, the isomorphism given by $x \mapsto \bar{x}$. Let $Q$ denote the ideal of $T^{*}(A \oplus \bar{A})$ generated by $x \otimes y, \bar{x} \otimes \bar{y}, x \otimes \bar{y} \otimes z-\langle x y z\rangle, \bar{x} \otimes y \otimes \bar{z}-\overline{\langle z y x\rangle}$ for $x, y, z \in A$. Set $U(A):=T^{*}(A \oplus \bar{A}) / Q$ and $\iota: x \mapsto x+Q$. Observing the elementary properties of the tensor product one notes the existence of an involution $j$ of $U(A)$ with $j(x+Q)=\bar{x}+Q$. Thus $(U(A), \iota, j)$ is a universal $j$-imbedding of $A$. For the 1st kind take $T^{*}(A)$ modulo the ideal $Q$ generated by the elements $x \otimes y \otimes z-\langle x y z\rangle$.

The proof of the second part of the statement is standard.

In the subsequent we may suppose that $\iota$ is the inclusion map. For an algebra $C$ let $C^{[n]}$ denote the $n$-fold cartesian product. Let $U(A)$ design a universal ( $j$-) imbedding of $A$. Obviously $U(A)$ is direct.

For a direct imbedding $B=A \oplus A \circ A$ let $\varepsilon_{1}: B \rightarrow A, \varepsilon_{2}: B \rightarrow A \circ A$ be 
the canonic projections. If $B$ is a direct $j$-imbedding let $\varepsilon_{1}, \varepsilon_{2}, \varepsilon_{-1}, \varepsilon_{-2}$ be its canonic projections onto $A, A \circ \bar{A}, \bar{A}, \bar{A} \circ A$. Set $B_{k}:=\varepsilon_{k}(B), k \in J:=\{1$, $2\}$, respectively $J:=\{-2,-1,1,2\}$. The formal multiplication of the $B$-components $B_{k}$ is obviously declared, e.g. for the 2 nd kind $A \cdot(\bar{A} \circ A):=A$, $(A \circ \bar{A}) \cdot(A \circ \bar{A}):=A \circ \bar{A}, \bar{A} \cdot \bar{A}:=\{0\}, \ldots$ Similar to the case of Lie triples [2] we will consider some homogeneous multilinear mappings of universal $(j-)$ imbeddings. For $x:=\left(x_{1}, \ldots, x_{n}\right) \in B^{[n]}$ with $x \in B_{k}, k=$ $k(i)$, the $B$-component is $\Phi(x):=\prod B_{k(i)}, i=1, \ldots, n$, the product formal. Let $C$ be a subalgebra of $B$ with $\varepsilon_{k}(C) \subset C$, and $B^{\prime}$ a $\left(j^{\prime}-\right)$ imbedding of $A^{\prime}$ with $A^{\prime}$ of the same kind as $A$. Then $f: C^{[n]} \rightarrow B^{\prime}$ is isovarying if

$$
\Phi(x) \subset B_{k} \Rightarrow f(x) \subset B_{k}^{\prime},
$$

and antivarying if for $\Phi(x) \neq\{0\}$

$$
\Phi(x) \subset B_{k} \Rightarrow f(x) \subset \bigoplus B_{l}^{\prime}, \quad l \neq k, l \in J .
$$

Any $f$ has a unique decomposition $f=f_{1}+f_{2}, f_{1}$ isovarying and $f_{2}$ antivarying. Denote $\varepsilon: f \mapsto f_{1}$. Then $\varepsilon^{2}:=\varepsilon \cdot \varepsilon=\varepsilon$.

Let $B:=A+M$, and let $J$ be the ideal in $U(B)$ equal to

$$
\{a \in B \circ M+M \circ B \mid a \circ A=A \circ a=\{0\}\},
$$

respectively in the case of the 2 nd kind

$$
\begin{aligned}
\{a \in B \circ \bar{M}+M \circ \bar{B}+\bar{B} \circ M+\bar{M} \circ B \mid a \circ(A+\bar{A}) & \\
= & (A+\bar{A}) \circ a=\{0\}\} .
\end{aligned}
$$

Then the universal module imbedding $M_{U}$ is the ideal of $U(A+M)$ generated by $M$, respectively $M \oplus \bar{M}$, in the case of 2nd kind associativity, modulo $J$. The maps corresponding to $\varepsilon$ and $\varepsilon, j$ for the 2 nd kind after the passages from $U(A+M)$ to $U(A)$ and $M_{U}$ are denoted equally. $M_{U}$ is an associative bimodule over $U(A)$ in the obvious way.

3. The cohomology. Let $C^{n}$ be the linear space of the $n$-cochains of $U(A)$ for $M_{U}$ [3], $n \in \mathbf{N}_{0}$. These are the $n$-linear mappings of $U(A)^{[n]}$ into $M_{U}$ for $n \in \mathbf{N}$, while $C^{0}=M_{U}$. The coboundary operator is defined by

$$
\begin{aligned}
\delta f\left(a_{1}\right) & =a_{1} \cdot f-f \cdot a_{1}, \quad \text { if } f \in C^{0}, \\
\delta f\left(a_{1}, \ldots, a_{n+1}\right)= & a_{1} \cdot f\left(a_{2}, \ldots, a_{n+1}\right) \\
& +\sum_{i=1}^{n}(-1)^{i} f\left(a_{1}, \ldots, a_{i} \circ a_{i+1}, \ldots, a_{n+1}\right) \\
& +(-1)^{n+1} f\left(a_{1}, \ldots, a_{n}\right) \cdot a_{n+1} \\
& \text { for } f \in C^{n}, n \neq 0, a_{i} \in U(A) .
\end{aligned}
$$

$Z^{n}, B^{n}$ denote the $K$-modules of the $n$-cocycles and the $n$-coboundaries where $B^{0}:=\{0\} . H^{n}\left(U(A), M_{U}\right):=Z^{n} / B^{n}$ is the $n$-dimensional cohomology group. If $x=\left(x_{1}, \ldots, x_{n}\right) \in U(A)^{[n]}, n \neq 0, U(A)$ a $j$-imbedding, set $j(x)$ : = $\left(j\left(x_{n}\right), \ldots, j\left(x_{1}\right)\right)$. For $f \in C^{0}$ extend $\varepsilon$ by $\varepsilon(f):=\varepsilon_{2} f$ if $A$ is of the 1st kind, and if $A$ is of the 2 nd kind let $\varepsilon(f):=\left(\varepsilon_{2}+\varepsilon_{-2}\right) f, f j:=f$. Then the $K$-module 
of the $n$-cochains of the associative triple system $A$ for $M$ is

$$
C^{n}(A, M):=\left\{f \in C^{n} \mid \varepsilon(f)=f\right\}
$$

for $n \in \mathrm{N}_{0}$ if $A$ and $M$ are associative of the 1st kind. In case of the 2 nd kind define

$$
C^{n}(A, M):=\left\{f \in C^{n} \mid \varepsilon(f)=f, j f j=(-1)^{n(n+1) / 2+1} f\right\}
$$

for $n \in \mathbf{N}_{0}$ if $\frac{1}{2} \in K$. If $2 \cdot K=\{0\}$ modify this definition by $C^{0}(A, M):=\{f$ $\in C^{0} \mid \varepsilon(f)=f, j f=f+z$ with $z \in M_{U}, a \cdot z=z \cdot a$ for all $\left.a \in U(A)\right\}$.

The coboundary operator $\partial$ is the restriction of $\delta$ from $C^{n}$ onto $C^{n}(A, M)$. The modules $C^{n}(A, M)$ together with $\partial$ define a cochain complex. $Z^{n}(A, M)$ is the submodule of $n$-cocycles of $C^{n}(A, M), B^{n}(A, M)$ that of $n$-coboundaries. Let $H^{n}(A, M)$ be the n-dimensional cohomology group with $H^{n}(A, M):=$ $Z^{n}(A, M) / B^{n}(A, M)$. Set $Z_{+}^{n}:=Z^{n} \cap C^{n}(A, M)$, and let $B_{+}^{n}:=B^{n} \cap$ $C^{n}(A, M)$. We have

LEMMA 1. Let $A$ be an associative triple system, $M$ an associative trimodule over $A$, and $\frac{1}{2} \in K$ in case of the 2 nd kind. Then

$$
H^{n}(A, M)=Z_{+}^{n} / B_{+}^{n} \text {, and } H^{n}\left(U(A), M_{U}\right) \cong H^{n}(A, M) \oplus H(n),
$$

$H(n)$ a submodule of $H^{n}\left(U(A), M_{U}\right), n \in \mathbf{N}_{0}$.

Proof. Let $A$ and $M$ be associative of the 2nd kind, and $\frac{1}{2} \in K$. Then for $f \in C^{n}$ we have $f=\varepsilon(f)+(f-\varepsilon(f))$ and $f=\frac{1}{2} \cdot((f+j f j)+(f-j f j))$. These are the decompositions of $f$ into eigenfunctions for the endomorphisms $\varepsilon$ and $\tilde{j}: f \mapsto j f j$. An easy verification shows that $\varepsilon$ and $\tilde{j}$ commute. Thus there is a weight space decomposition $C^{n}=\bigoplus C_{\alpha, \beta}^{n}, \alpha$ the root of $\varepsilon, \beta$ the root of $\tilde{j}$ for $C_{\alpha, \beta}^{n}$. Now $C^{n}(A, M)=C_{1,(-1)^{n(n+1) / 2+1}}^{n}$. Since $\delta: C_{\alpha, \beta}^{n-1} \rightarrow C_{\alpha, \beta^{\prime}}^{n}, \beta^{\prime}=\beta$. $(-1)^{n}$, we get $B^{n}(A, M)=B_{+}^{n}$. The rest is obvious. For the case of the 1st kind one proceeds similarly and even more immediately.

The associative triple $A$ is separable over the field $K$, if for every base field extension $\tilde{K}$ of $K$ the base field extension $\tilde{A}$ of $A$ is semisimple. Note that as in the binary case the radical of a ternary algebra $A$ coincides with the radical of the ternary ring $A$ when $\frac{1}{2} \in K$ [8]. We receive

COROLlaRY 1. Let $M$ be an associative trimodule of the 1 st or 2 nd kind over $A$, and $A$ separable over a field $K, \operatorname{char}(K) \neq 2$. Then for all $n \in \mathbf{N}, H^{n}(A, M)$ $=\{0\}$.

Proof. Because $A$ is semisimple the standard imbedding of $A$ is semisimple too by [6], respectively [7]. Any semisimple imbedding, respectively $j$-imbedding, is isomorphic to $U(A)$. The isomorphism follows as the corresponding one in case of Lie triples [4]. Thus $U(A)$ is separable and

$$
H^{n}\left(U(A), M_{U}\right)=\{0\} \quad \text { for all } n \in \mathbf{N} \text {. }
$$

Corollary 1 includes the statements corresponding to the two Whitehead lemmas.

A linear mapping $D: A \rightarrow M$ is a derivation of $A$ in $M$ if

$$
D(\langle x y z\rangle)=\langle D(x) y z\rangle+\langle x D(y) z\rangle+\langle x y D(z)\rangle, \quad x, y, z \in A .
$$


Inner derivations are those generated by the triple multiplications in the semidirect sum $A+M$.

If $\operatorname{Der}(A, M)$ is the $K$-module of derivations of $A$ in $M$, then we have $Z^{1}(A, M) \cong \operatorname{Der}(A, M)$, the elements of $B^{1}(A, M)$ related to inner derivations of $A$ in $M$. As isomorphism of $Z^{1}(A, M)$ onto $\operatorname{Der}(A, M)$ we may choose the restriction of the elements $f \in Z^{1}(A, M)$ from $U(A)$ onto $A$. It follows immediately that in case of the 2 nd $\operatorname{kind}, \operatorname{char}(K)=2$ and $n=1$, Corollary 1 is valid too. Hence

Corollary 2. Let $A$ and $M$ be associative of the same kind, $A$ separable over the field $K$, and $\operatorname{char}(K) \neq 2$ in case of the 1 st kind. Then every derivation of $A$ in $M$ is inner.

We may remark that in case of $\operatorname{char}(K)=2$ the trivial 1st kind triple $A:=\{0,1\}$ with $\langle 111\rangle=1$ is separable, while the radical of $U(A)$ is $\{0$, $1+1 \circ 1\} \neq\{0\}$.

Now let $C$ denote a binary algebra, $N$ a $C$-bimodule. A semiautomorphism $f$ of $N$ is an algebra automorphism of the semidirect sum $C+N$ with $f(A) \subset$ $A, f(N) \subset N$. It holds:

LEMMA 2. Let $G$ be a finite group of semiautomorphisms of a bimodule $N$ over an algebra $C, n$ the order of $G$, and $1 / n \in K$. If $N=Q \oplus P, Q$ and $P$ are $C$-submodules with $G(Q)=Q$, then there exists a $C$-submodule $R$ of $N$ with $G(R)=R$ and $N=Q \oplus R$.

The proof follows considering the semidirect sum $C+N$ and going on along the lines of [10].

Irreducible trimodules, complete reducibility, triple (trimodule) homomorphisms are defined as obvious. If $A$ is semisimple, $Z$ the center of $U(A)$, and $Z \otimes Z$ semisimple, $A$ is c-semisimple. Denote $W:=U(A) \otimes U(A)^{\mathrm{op}}, U(A)^{\mathrm{op}}$ the opposite algebra for $U(A)$. For $c$-semisimple $A, W$ is semisimple. For a direct imbedding $B$ let $\sigma$ be the natural algebra automorphism with $\sigma(x)=-x$ and fixing $x \circ y$ when $x, y \in A$.

Proposition 1. Let $M$ be an associative trimodule over $A, K \cdot a$ field with $\operatorname{char}(K) \neq 2$, and $A$ c-semisimple. Then $M$ is completely reducible over $K$.

Proof. $M_{U}$ is an associative left $W$-module by $x \otimes y \cdot m=x \circ m \circ y$ with $x, y \in U(A), m \in M_{U}$. Then $M_{U}=e \circ M_{U} \circ e \oplus M_{0}$, $e$ the unit of $U(A)$, $M_{0}$ the maximal zero $W$-submodule, is a decomposition into $W$-submodules. Set $M:=e \circ M_{U} \circ e$. It may be noted that for the 2nd kind $e=e_{1}+e_{2}$ with $e_{1} \in A \circ \bar{A}, e_{2} \in \bar{A} \circ A$, so that $\varepsilon_{1}(M)=e_{1} \circ M_{U} \circ e_{2}$. Thus $M=\varepsilon_{1}(M) \oplus$ $\varepsilon_{1}\left(M_{0}\right)$ is a decomposition of $M$ into subtrimodules. Consider now $\varepsilon_{1}\left(M_{0}\right)$ as a left- and a right-module over the semisimple algebra $A \circ A$ over which it decomposes into irreducible submodules [6], and equally for $A \circ \bar{A} \oplus \bar{A} \circ A$ in case of the 2 nd kind. Hence $\varepsilon_{1}\left(M_{0}\right)$ is completely reducible over $A$.

Set $G:=\{$ Id, $\sigma\}$ for the lst kind. Then $G(M) \subset M$. If $M \neq\{0\}, M$ decomposes by Lemma 2 or [5, p. 117] into minimal $G$-invariant submodules $P_{k} \neq\{0\}$ over $W, k=1, \ldots, t$. Therefore $\varepsilon_{1}\left(P_{k}\right) \subset P_{k}$. Hence $N_{k}:=\varepsilon_{1}\left(P_{k}\right)$ is an irreducible subtrimodule. Thus $M$ is a completely reducible $A$-trimodule. 
The proposition says equivalently that under its conditions on $A$ any short exact sequence of associative $A$-trimodules of the same kind, the connecting mappings being trimodule homomorphisms, splits by a trimodule homomorphism.

An extension of the associative triple $A$ by $M$ is a short exact sequence of associative triples $M, E, A$ of the same kind $\{0\} \rightarrow M \rightarrow^{e} E \rightarrow^{p} A \rightarrow\{0\}$ with $e, p$ triple homomorphisms. $M$ is in a canonic way a trimodule over $A$. Let ${ }^{(1)} M:=\langle M M A\rangle+\langle M A M\rangle+\langle A M M\rangle$. The extension is singular if ${ }^{(1)} M=$ $\langle M M M\rangle=\{0\}$. The extension is split or trivial if there exists a triple homomorphism $q: A \rightarrow E$, with $p q=\operatorname{Id}_{A}$. The extension $\{0\} \rightarrow M \rightarrow^{f} E^{\prime}$ $\rightarrow^{q} A \rightarrow\{0\}$ is equivalent to the first if there is a triple homomorphism $k$ : $E \rightarrow E^{\prime}$ so that the diagram

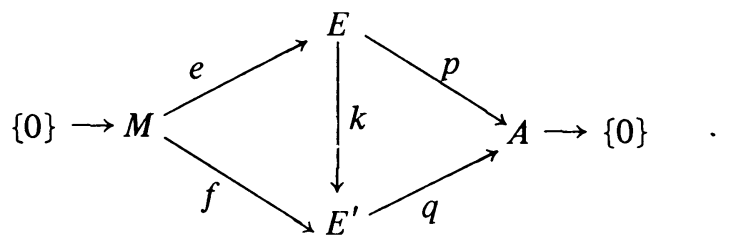

is commutative. Then $k$ is an isomorphism. Let $J^{\prime}$ be the ideal of $U(E)$ defined as $J$, substituting $e(M), E$ for $M$ and $A+M$. Set $U(E)^{\prime}:=$ $U(E) / J^{\prime}$. Design the maps induced by $\varepsilon$ and $j$ on $U(E)^{\prime}$ equally. For a singular extension there is a commutative diagram:

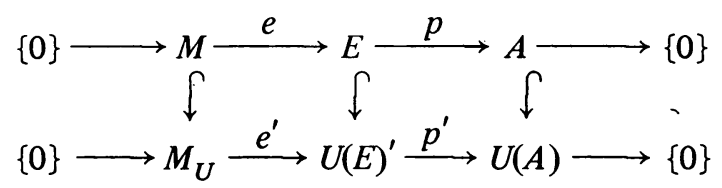

$e^{\prime}, p^{\prime}$ algebra homomorphisms invariant relative to $\varepsilon$ and respectively for $j$. Note that the second row is also exact (cf. [2, pp. 158-159]).

A factor set $f$ of $A$ in $M$ is, as usual, a trilinear mapping $f: A \times A \times A \rightarrow$ $M$ so that the $K$-module direct sum $A+M$ with $[x y z]:=\langle x y z\rangle+f(x, y, z)$, ${ }^{(1)} M:=\{0\},\langle M M M\rangle:=\{0\}$, and the trimodule structure of $M$, is an associative triple of the same kind. $f$ is trivial if there is $s: A \rightarrow M, s K$ - linear with

$$
f(x, y, z):=s(\langle x y z\rangle)-\langle s(x) y z\rangle-\langle x s(y) z\rangle-\langle x y s(z)\rangle .
$$

Let $A$ be $K$-projective in the usual sense. Then any extension of the triple $A$ is split for the $K$-module $A$. Similarly as for algebras, the classes of equivalent singular extensions of $A$ by $M$ constitute a $K$-module isomorphic to the $K$-module of factor sets, the trivial factor sets corresponding to the classes of the split extensions. One then obtains, arguing as [2, pp. 159-160] for Lie triples,

THEOREM 2. If $A$ and the trimodule $M$ over $A$ are associative of the same kind, $A K$-projective, and $\frac{1}{2} \in K$ in case of the 2 nd kind, then there is a module isomorphism $H^{2}(A, M) \cong E(A, M) / E_{t}(A, M), E(A, M)$ the $K$-module of the classes of equivalent singular extensions of $A$ with kernel $M, E_{t}(A, M)$ the submodule of the classes of split extensions. 
If $A^{(1)}:=\langle A A A\rangle, A^{(n+1)}=\left(A^{(n)}\right)^{(1)}, n \in \mathrm{N}$, then $A$ is solvable when $A^{(m)}=$ $\{0\}$ for some $m$. Set for an ideal $I$ in $A,{ }^{(n+1)} I:={ }^{(1)}\left({ }^{(n)} I\right) . I$ is $S$-solvable if for one $m,{ }^{(m)} I=\{0\}$. Because $R:=\operatorname{Rad}(A) \subset \operatorname{Rad}(U(A)), R$ is solvable if $K$ is a field. We have for arbitrary $K$

Lemma 3. Let I be an ideal of $A$. Then:

(a) $I$ is solvable exactly if it is $S$-solvable.

(b) When $I$ is solvable, $I \neq\{0\}$, then ${ }^{(1)} I \varsubsetneqq I$.

Proof. (a) From $B^{(1)} \subset^{(1)} B$ we get $B^{(n)} \subset^{(n)} B$ for any $n \in \mathbf{N}$. Further one verifies ${ }^{(3)} B \subset B^{(1)}$. Thus ${ }^{(3 n)} B \subset B^{(n)}$. Hence we get (a). (a) $\Rightarrow(\mathrm{b})$.

The proof of the Wedderburn principal theorem reduces by Lemma 3 to the singular case. For associative triples of the 2 nd kind the statement is implied by [1]. On behalf of Corollary 1 it follows:

COROllary 3. Let $A$ be a finite dimensional associative triple system over a field $K, R$ the radical, $A / R$ separable, and $\operatorname{char}(K) \neq 2$. Then $A$ decomposes $A=B \oplus R, B$ a subtriple of $A, B \cong A / R$.

\section{REFERENCES}

1. R. Carlsson, Der Wedderburnsche Hauptsatz für alternative Paare (to appear).

2. B. Harris, Cohomology of Lie triple systems and Lie algebras with involution, Trans. Amer. Math. Soc. 98 (1961), 148-162. MR 22 \#11068.

3. G. Hochschild, On the cohomology groups of an associative algebra, Ann. of Math. (2) 46 (1945), 58-ó7. MR 6, 114.

4. N. Jacobson, General representation theory of Jordan algebras, Trans. Amer. Math. Soc. 70 (1951), 509-530. MR 12, 797.

5. __ Structure of rings, rev. ed., Amer. Math. Soc. Colloq. Publ., vol. 37, Amer. Math. Soc., Providence, R. I., 1964. MR 36 \# 5158.

6. W. G. Lister, Ternary rings, Trans. Amer. Math. Soc. 154 (1971), 37-55. MR 42 \#7716.

7. O. Loos, Assoziative Tripelsysteme, Manuscripta Math. 7 (1972), 103-112. MR 46 \#3581.

8. H. C. Myung, A characterization of the Jacobson radical in ternary algebras, Proc. Amer. Math. Soc. 38 (1973), 228-234. MR 49 \#363.

9. P. Seibt, Cohomology of algebras and triple systems, Comm. Algebra 3 (1975), 1097-1120.

10. E. J. Taft, Cleft algebras with operator groups, Portugal. Math. 20 (1961), 195-198. MR 25 \#2103.

Universität Hamburg, Mathematisches Seminar, Bundesstrasse 55,2 Hamburg 13, FEDERAL REPUBLIC OF GERMANY 